\title{
Thermally activated escape rate for the Brownian motion of a fixed axis rotator in an asymmetrical double-well potential for all values of the dissipation
}

\author{
Yuri P. Kalmykov ${ }^{\text {a) }}$ \\ Groupe de Physique Moléculaire, MEPS, Université de Perpignan, 52, Avenue Paul Alduy, \\ 66860 Perpignan Cedex, France \\ Sergey V. Titov \\ Institute of Radio Engineering and Electronics, Russian Academy of Sciences, Vvedenskii Square 1, \\ Fryazino, Moscow Region, 141190, Russian Federation \\ William T. Coffey \\ Department of Electronic and Electrical Engineering, Trinity College, Dublin 2, Ireland
}

(Received 8 June 2005; accepted 30 June 2005; published online 7 September 2005)

\begin{abstract}
The Kramers theory of the escape rate of a Brownian particle from a potential well as extended by Mel'nikov and Meshkov, [J. Chem. Phys. 85, 1018 (1986)] is used to evaluate the relaxation times and the dynamic susceptibility for the rotational Brownian motion of fixed axis rotators in an asymmetric double-well potential. An expression for the escape rate valid for all values of the dissipation including the very low damping (VLD), very high damping (VHD), and crossover regimes is derived. It is shown that this expression provides a good asymptotic estimate of the inverse of the smallest nonvanishing eigenvalue $\lambda_{1}$ of the underlying Fokker-Planck operator calculated by using the matrix-continued fraction method. For low barriers, where the Mel'nikov and Meshkov approach is not applicable, analytic equations for the correlation time $\tau_{\|}$of the longitudinal dipole correlation function in the VLD and VHD limits are derived and a simple extrapolating equation valid for all values of the damping is proposed. (C) 2005 American Institute of Physics. [DOI: 10.1063/1.2008250]
\end{abstract}

\section{INTRODUCTION}

The Brownian motion in an asymmetric periodic potential is of interest in the solution of a variety of physical problems involving a relaxation process. Among the most prominent of these are dielectric relaxation and the dynamic Kerr effect of nematic liquid crystals, magnetic relaxation of single-domain ferromagnetic particles, dynamic response of Josephson tunneling junctions, transport phenomena in semiconductors, etc. $^{1-3}$

One of the most important characteristics associated with the Brownian motion in any asymmetric multiwell potential is the dependence of the greatest relaxation time and the integral relaxation time on the asymmetry parameter. In this context we remark that by taking longitudinal dielectric relaxation in a symmetric double-well potential as an example the greatest relaxation time which is the time required to escape the well or the relaxation time of the longest-lived relaxation mode is accurately approximated by the integral relaxation time $\tau_{\|}$. The integral relaxation time is the area under the decay curve of the electric polarization following the removal of a steady field and corresponds in linear response to the correlation time of the dipole moment. On the other hand, in an asymmetric double-well potential for values of the asymmetry parameter well below that required to destroy the double-well nature of the potential the integral

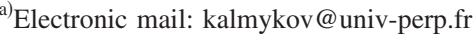

relaxation time may diverge exponentially from the greatest relaxation time with consequent suppression of the overbarrier relaxation mode so that the low-frequency dielectric loss associated with this mode effectively disappears. This phenomenon of course does not occur in symmetric potentials. Hence it is of some importance to establish the effect of the asymmetry parameter on the relaxation behavior. The behavior as a function of that parameter has been studied both numerically and analytically when the inertial of a dipole is ignored. ${ }^{4,5}$ However, no calculations have been carried out for asymmetric potentials when inertial effects are included so that the very high-frequency modes associated with the librational motion in the wells of the potential have been excluded.

In the present context we remark that the greatest relaxation time is essentially the inverse of the smallest nonvanishing eigenvalue $\lambda_{1}$ of the characteristic equation or secular determinant of the relevant dynamical system. In other words $\lambda_{1}^{-1}$ is the lifetime of the longest-lived relaxation mode of the system. The greatest relaxation time may also be obtained by calculating the mean first passage times from each of the wells of the potential. ${ }^{6}$ As far as the calculation of $\lambda_{1}$ is concerned, the secular equation may be generated by averaging the appropriate Langevin equation over its realizations in phase space yielding the hierarchy of differential-recurrence equations governing the decay functions of the system. Alternatively, one may expand the solution of the associated probability density diffusion equation (usually the special- 
ized form of the Fokker-Planck equation applicable to separable and additive Hamiltonians comprising the sum of the potential and kinetic energies known as the Klein-Kramers equation) in Fourier series in the position and velocity variables. ${ }^{1,2}$ In each of the two methods, the secular determinant results from truncation of the set of differentialrecurrence relations at a number large enough to achieve convergence of the resulting set of simultaneous ordinary differential equations. Alternatively, if the problem is represented in the frequency domain so that the more powerful continued fraction method may be used (more powerful in the sense that it is very effective from a computational point of view), many convergents must be taken. ${ }^{1,7}$ Thus, $\lambda_{1}$ is not in general available in closed form as it is always rendered as the smallest root of a high-order polynomial equation. Hence, it is difficult to compare $\lambda_{1}$ so determined with experimental observations of the greatest relaxation time or the relaxation rate. Fortunately (noting that $\lambda_{1}$ for sufficiently high barriers has exponential dependence on the barrier height), a way of overcoming this difficulty is to utilize an ingenious method originally proposed by Kramers ${ }^{8}$ in connection with thermally activated escape of particles out of a potential well. His idea, motivated by the fluctuationdissipation theorem, ${ }^{2}$ is to calculate the prefactor $A$ in an Arrhenius-type equation for the reaction rate $\Gamma$, viz.,

$$
\Gamma=A \frac{\omega_{a}}{2 \pi} e^{-\Delta V / k T}
$$

Here $\Delta V$ is the height of the barrier, $k$ is Boltzmann's constant, and $T$ is the temperature. The parameter $A$ represents the interchange of energy between the reacting particles and their surroundings or heat bath (for reviews of applications of Kramers' method, see Refs. 9 and 10). The frequency $\omega_{a} / 2 \pi$ which is the frequency of oscillation of a particle in the potential well with a minimum at $a$ is called the attempt frequency. The original Arrhenius equation which is obtained when $A=1$ [corresponding to transition state theory (TST)] assumes perpetual thermal equilibrium everywhere at temperature $T$. Thus no account is taken of nonequilibrium effects due to the leaking of particles over the potential barrier at $c$. In reality the Maxwell-Boltzmann distribution no longer holds in the vicinity of the transition state $c$ because the fluctuation-dissipation theorem describing the coupling of the reacting particles to their surroundings or heat bath is violated by the Arrhenius equation.

Kramers ${ }^{8}$ overcame the problem of the departure from equilibrium by obtaining asymptotic solutions for $\lambda_{1}$ which in this context is termed as the Kramers escape rate from the Klein-Kramers equation in the limits of very small and intermediate to high dissipative coupling to the bath. These solutions, which are valid for high barriers $(\Delta V \gg k T)$ so that the concept of an escape rate is valid, provide closed-form expressions for the escape rate and its inverse the greatest relaxation time $\tau \approx \lambda_{1}^{-1}$ which may be easily compared with the experiment. Kramers was, however, unable to find asymptotic solutions for the crossover regime. This problem, named the Kramers turnover problem, was solved nearly 50 years later by Mel'nikov ${ }^{11}$ and Mel'nikov and Meshkov. ${ }^{12}$ They gave an integral formula bridging the very low damp- ing (VLD) and TST solutions. Thus, they postulate from heuristic reasoning, essentially appealing to continuity that an escape rate formula valid for all values of the damping may be given by simply multiplying the general intermediate-tohigh damping (IHD) result by their bridging integral. Mel'nikov $^{11}$ has further extended the bridging integral method to take into account quantum effects in a semiclassical way. Furthermore, Grabert ${ }^{13}$ and Pollak et al. ${ }^{14}$ later presented a complete solution of the Kramers turnover problem and have shown that the Mel'nikov and Meshkov universal formula can be obtained without ad hoc interpolation between the weak and strong damping regimes. In the semiclassical limit, the latter theory was extended to the quantum regime by Rips and Pollak. ${ }^{15}$

As far as the verification of the universal turnover formula of Mel'nikov and Meshkov is concerned, very few calculations based on either the exact solutions of the KleinKramers equation or on numerical simulations of the Brownian dynamics have ever been given. Exceptions are the comparison of the universal turnover formulas with the numerical results for the escape from a single well, which were given in Refs. 16 and 17 and the study of the onedimensional translational Brownian motion in a periodic potential undertaken by Ferrando et al. ${ }^{18}$ Another exception is the treatment of the same one-dimensional problem and its generalization to diffusion on a surface which was accomplished by Pollak and co-workers in Refs. 19-21, respectively. Examples of the exact treatment of rotational Brownian motion problems are even fewer. Pastor and Szabo ${ }^{22}$ tested the Mel'nikov-Meshkov formula in the context of orientational relaxation for a linear molecule in a uniaxial potential and Coffey et al. ${ }^{23}$ considered a two-dimensional ana$\log$ of that model (planar rotation in a double-well cosine potential). Also Coffey and co-workers ${ }^{10,24,25}$ extended the Mel'nikov-Meshkov calculation to magnetization relaxation of single-domain ferromagnetic particles with nonaxially symmetric potentials of the magnetocrystalline anisotropy.

In the context of the present problem of relaxation in asymmetrical potential wells we remark that the majority of the foregoing results have been obtained for potentials with equivalent wells. There the symmetry of the potential masks interesting effects, e.g., the exponential divergence of the integral relaxation time and the greatest relaxation time which may appear for potentials with nonequivalent wells, so that the asymmetry of the potential can radically alter the characteristics of the relaxation process. ${ }^{2}$ Exact numerical and accurate analytical solutions of this problem have been obtained in the very high damping limit when the inertial effects of the relaxing population may be ignored. However, it appears that solutions valid for all values of the damping for an asymmetric potential have not yet been obtained. Here we demonstrate that the Mel'nikov and Meshkov approach when applied to the particular problem of the inertial Brownian motion of a fixed axis rotator in a asymmetric doublewell potential yields an accurate solution for the greatest relaxation time $\lambda_{1}^{-1}$ for high barriers and for all values of the dissipation. Such a potential allows the flipping of rotators to neighboring wells, thus permitting both relaxation and oscillatory behavior in the same model and so may simulta- 
neously explain both the low-frequency relaxation and farinfrared absorption spectra of dipolar systems. ${ }^{2,26}$ Various applications of this model are discussed in detail in Refs. 2 and 26. The detailed description of the model for a symmetric double-well potential is given in Ref. 23. Here, we calculate the escape rate by the Mel'nikov-Meshkov asymptotic (in the sense that it applies for high barriers) and universal (in the sense that it is valid for all values of the damping) formula for an asymmetrical potential. Then we compare the inverse escape rate with the greatest relaxation time evaluated from the exact matrix-continued fraction solution of the underlying Langevin equation. Applications of the Mel'nikov-Meshkov asymptote to the estimation of the integral relaxation time (in the present context where linear response is assumed as the correlation time) of the longitudinal relaxation function, the complex susceptibility, etc. are also discussed and exact solutions for the electric susceptibility valid in all frequency ranges are presented. Whence one may conclude that essentially three relaxation-time processes exist: (i) a slow overbarrier relaxation process with relaxation time given by $\lambda_{1}^{-1}$ which gives rise to low-frequency absorption with an Arrhenius-type relaxation time; (ii) relatively fast near degenerate decay modes in the wells of the potential which may be approximated by a single decay mode and give rise in the frequency domain to weak intermediate frequency absorption; and (iii) high-frequency oscillatory modes which produce absorption in the far-infrared band of frequencies. These three relaxation modes are obviously present in symmetric potentials also. However, introducing asymmetry will cause at a certain critical value of the asymmetry parameter the overbarrier mode to become so weak that it will be almost completely suppressed while both intrawell and oscillatory modes remain.

\section{BASIC RELATIONS}

Our starting point is the Langevin equation for a dipole $\boldsymbol{\mu}$ rotating about an axis normal to the plane of rotation, ${ }^{2}$

$$
I \ddot{\phi}(t)+\zeta \dot{\phi}(t)+\frac{d V(\phi)}{d \phi}=\lambda(t)
$$

where $I$ is the moment of inertia of a rotator about the axis of rotation, $\phi$ is the angle specifying the orientation of a rotator, and $\zeta \dot{\phi}(t)$ and $\lambda(t)$ are the frictional and random torques acting on a rotator due to the Brownian motion arising from the heat bath. It is assumed that the random torque $\lambda(t)$ has the white-noise properties

$$
\overline{\lambda(t)}=0, \quad \overline{\lambda(t) \lambda\left(t^{\prime}\right)}=2 k T \zeta \delta\left(t-t^{\prime}\right) .
$$

Here the overbar denotes the statistical average over a large number of rotators which have at time $t$ identical angular velocity $\dot{\phi}$ and identical angular position $\phi$. In accordance with the notation of Refs. 1 and 2, we shall use the notation $\phi(t)$ and $\dot{\phi}(t)$ to denote the random variables while we shall denote their sharp (definite) values or realizations at time $t$ by $\phi$ and $\dot{\phi}$. The internal field due to molecular interactions and the external field are represented by the (periodic) double-well potential (see Fig. 1),

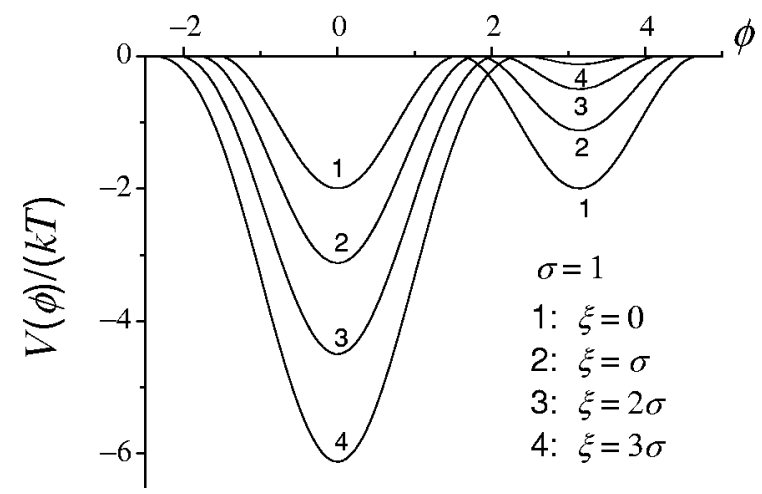

FIG. 1. Potential $V(\phi) /(k T)=-2 \sigma \cos ^{2} \phi-\xi \cos \phi-\xi^{2} /(8 \sigma)$ for $\sigma=1$ and various values of $\xi$.

$$
\begin{aligned}
V(\phi) /(k T) & =-2 \sigma \cos ^{2} \phi-\xi \cos \phi-\xi^{2} /(8 \sigma) \\
& =-2 \sigma(\cos \phi+h)^{2},
\end{aligned}
$$

where $\sigma=V_{0} /(k T)$ is the barrier height parameter, $\xi=\mu E /(k T)$ is the external field parameter, $E$ is the external field, $h=\xi /(4 \sigma)$, and $k T$ is the thermal energy. The corresponding Klein-Kramers (Fokker-Planck) equation for the distribution function $W(\phi, \dot{\phi}, t)$ can be written as ${ }^{2}$

$$
\dot{W}=L_{\mathrm{FP}} W,
$$

where the Fokker-Planck operator $L_{\mathrm{FP}}$ is given by ${ }^{26}$

$$
L_{\mathrm{FP}} W=-\dot{\phi} \frac{\partial W}{\partial \phi}+\frac{1}{I} \frac{d V}{d \phi} \frac{\partial W}{\partial \dot{\phi}}+\beta\left(\frac{\partial}{\partial \dot{\phi}}(\dot{\phi} W)+\frac{k T}{I} \frac{\partial^{2} W}{\partial \dot{\phi}^{2}}\right)
$$

and $\beta=\zeta / I$. The first two terms on the right-hand side of Eq. (5) comprise the convective or Liouville term describing in the absence of dissipation the undamped streaming motion along the energy trajectories in phase space corresponding to Hamilton's equations. The last term (the diffusion term) represents the interchange of energy (dissipative coupling) with the heat bath.

In orientational relaxation, the quantity of greatest interest is the equilibrium correlation function $C(t)$ of the longitudinal component of the dipole moment defined as

$$
C(t)=\frac{\langle\cos \phi(0) \cos \phi(t)\rangle_{0}-\langle\cos \phi(0)\rangle_{0}^{2}}{\left\langle\cos ^{2} \phi(0)\right\rangle_{0}-\langle\cos \phi(0)\rangle_{0}^{2}}
$$

(the angular brackets denote the equilibrium ensemble average). This allows one to calculate the longitudinal complex susceptibility $\chi(\omega)=\chi^{\prime}(\omega)-i \chi^{\prime \prime}(\omega)$ defined as ${ }^{2}$

$$
\frac{\chi(\omega)}{\chi^{\prime}(0)}=1-i \omega \widetilde{C}(\omega)
$$

where $\chi^{\prime}(0)=\left(\mu^{2} N_{0} / k T\right)\left(\left\langle\cos ^{2} \phi(0)\right\rangle_{0}-\langle\cos \phi(0)\rangle_{0}^{2}\right)$ is the static susceptibility and $N_{0}$ is the number of dipoles per unit 
volume. Moreover, one can also calculate the correlation time $\tau_{\|}$which is defined as the area under the curve of $C(t)$ so that

$$
\tau_{\|}=\int_{0}^{\infty} C(t) d t=\tilde{C}(0) .
$$

The time $\tau_{\|}$may equivalently be defined in terms of the eigenvalues $\left(\lambda_{k}\right)$ of the Fokker-Planck operator $L_{F P}$ from Eq. (5) because the function $C(t)$ may be formally written as the discrete set of relaxation modes ${ }^{1,2}$

$$
C(t)=\sum_{k} c_{k} e^{-\lambda_{k} t}
$$

Thus from Eqs. (8) and (9) one has

$$
\tau_{\|}=\sum_{k} c_{k} / \lambda_{k}
$$

where $\Sigma_{k} c_{k}=1$ so that $\tau_{\|}$contains contributions from all the eigenvalues $\lambda_{k}$. In general, for nonequivalent wells above a critical value of the asymmetry parameter, $\tau_{\|}$may differ exponentially from the inverse of the smallest nonvanishing eigenvalue $\lambda_{1}^{-1}$, which is the longest relaxation time $\tau$ of the system. ${ }^{2,4}$ In the frequency domain of representation of the relaxation process, $\lambda_{1}$ corresponds to the half-width of the spectrum $\tilde{C}(\omega)$ or, equivalently, to the low-frequency maximum of the dielectric loss spectra $\chi^{\prime \prime}(\omega)$.

First, we present an exact method of solution of the Langevin equation (2) based on matrix-continued fractions and so exact evaluation of $\widetilde{C}(\omega), \tau_{\|}$, and $\lambda_{1}^{-1}$. Then we show how $\lambda_{1}^{-1}$ and the low-frequency part of $\widetilde{C}(\omega)$ can be evaluated in the low-temperature (high barrier) limit from the universal Mel'nikov-Meshkov equation.

\section{DIFFERENTIAL-RECURRENCE RELATIONS FOR THE CORRELATION FUNCTIONS}

We recall ${ }^{2}$ that the average value $\bar{\phi}$ of a random variable $\phi(t)$ at time $t$ is calculated from the Langevin equation (2) regarded as an integral equation and interpreted as a Stratonovich stochastic equation by expressing $\bar{\phi}$ as an equation of motion for a sharp value $\phi(t)=\phi$ at time $t$. Equation (2) then allows us to derive (see for details Ref. 2, Chap. 10) a hierarchy of recurrence equations for the equilibrium correlation functions $c_{n, q}(t)$ defined as

$$
\begin{aligned}
c_{n, q}(t)= & \left\langle\cos \phi(0) H_{n}[\eta \dot{\phi}(t)] e^{-i q \phi(t)}\right\rangle_{0} \\
& -\langle\cos \phi(0)\rangle_{0}\left\langle H_{n}[\eta \dot{\phi}(0)] e^{-i q \phi(0)}\right\rangle_{0},
\end{aligned}
$$

where $H_{n}(z)$ is the Hermite polynomial of order $n,{ }^{27} \phi(0)$ is the initial value of $\phi(t)$, and $\eta=\sqrt{I /(2 k T)}$. We have for the equations of motion of the sharp values that is the equation of motion of the Fourier coefficients of the Green function or fundamental solution of the problem

$$
\begin{aligned}
\eta \frac{d}{d t} H_{n}[\eta \dot{\phi}] e^{-i q \phi} \\
\quad=\overline{2 n H_{n-1}[\eta \dot{\phi}] e^{-i q \phi} \eta^{2} \ddot{\phi}-i q e^{-i q \phi} \eta \dot{\phi} H_{n}[\eta \dot{\phi}] .}
\end{aligned}
$$

The two terms on the right-hand side of Eq. (12) can be rearranged as

$$
\begin{aligned}
& 2 n \overline{H_{n-1}[\eta \dot{\phi}(t)] e^{-i q \phi(t)} \eta^{2} \ddot{\phi}(t)}=\overline{n e^{-i q \phi(t)} H_{n-1}[\eta \dot{\phi}(t)]\left[-2 \beta^{\prime} \eta \dot{\phi}(t)-2 \sigma \sin 2 \phi(t)-\xi \sin \phi(t)+\lambda(t) /(k T)\right]} \\
& =-i n \sigma\left[e^{-i(q+2) \phi}-e^{-i(q-2) \phi}+2 h\left(e^{-i(q+1) \phi}-e^{-i(q-1) \phi}\right)\right] H_{n-1}[\eta \dot{\phi}] \\
& -n \beta^{\prime} e^{-i q \phi}\left[H_{n}[\eta \dot{\phi}]+2(n-1) H_{n-2}[\eta \dot{\phi}]\right]+\overline{e^{-i q \phi(t)} H_{n-1}[\eta \dot{\phi}(t)] \lambda(t)} /(k T)
\end{aligned}
$$

and

$$
\begin{aligned}
-i \overline{e^{-i q \phi(t)} \eta \dot{\phi}(t) H_{n}[\eta \dot{\phi}(t)]}= & -i(q / 2) e^{-i q \phi}\left(H_{n+1}[\eta \dot{\phi}]\right. \\
& \left.+2 n H_{n-1}[\eta \dot{\phi}]\right),
\end{aligned}
$$

respectively. Here, we have noted that ${ }^{27}$

$$
\frac{d}{d z} H_{n}(z)=2 n H_{n-1}(z), \quad H_{n+1}(z)=2 z H_{n}(z)-2 n H_{n-1}(z),
$$

and $^{2}$

$$
\overline{e^{-i q \phi(t)} H_{n-1}[\eta \dot{\phi}(t)] \lambda(t)}=2 \beta^{\prime} k T(n-1) e^{-i q \phi} H_{n-2}[\eta \dot{\phi}],
$$

where $\beta^{\prime}=\beta \eta$ is the dimensionless damping parameter. Thus we have

$$
\begin{aligned}
\eta \frac{d}{d t} H_{n}[\eta \dot{\phi}] e^{-i q \phi}= & -n \beta^{\prime} e^{-i q \phi} H_{n}[\eta \dot{\phi}] \\
& -\frac{i q}{2} e^{-i q \phi}\left(H_{n+1}[\eta \dot{\phi}]+2 n H_{n-1}[\eta \dot{\phi}]\right) \\
& -i n \sigma\left[e^{-i(q+2) \phi}-e^{-i(q-2) \phi}\right. \\
& \left.+2 h\left(e^{-i(q+1) \phi}-e^{-i(q-1) \phi}\right)\right] H_{n-1}[\eta \dot{\phi}] .
\end{aligned}
$$

By multiplying Eq. (13) by $\cos \phi(0)$ and again averaging the equation so obtained by postulating the equilibrium (Maxwell-Boltzmann) distribution function of $\phi, \dot{\phi}$ at the instant $t=0$, we can derive (as described in detail in Ref. 2) differential-recurrence equations for the correlation functions $c_{n, q}(t)$, viz., 


$$
\begin{aligned}
\eta \frac{d}{d t} c_{n, q}(t)= & -n \beta^{\prime} c_{n, q}(t)-\frac{i q}{2}\left[c_{n+1, q}(t)+2 n c_{n-1, q}(t)\right] \\
& -i n \sigma\left[c_{n-1, q+2}(t)-c_{n-1, q-2}(t)\right] \\
& -2 i n \sigma h\left[c_{n-1, q+1}(t)-c_{n-1, q-1}(t)\right] .
\end{aligned}
$$

The solution of the recurrence in Eq. (14) can be obtained by a matrix-continued fraction method. ${ }^{1,2}$ We remark that exactly the same hierarchy may be obtained by first calculating Green's function of the Fokker-Planck equation (5) and then averaging the hierarchy over the equilibrium MaxwellBoltzmann distribution.

\section{MATRIX-CONTINUED FRACTION SOLUTION}

By Laplace transformation, we have from Eq. (14)

$$
\begin{aligned}
{\left[\eta s+n \beta^{\prime}\right] \widetilde{c}_{n, q}(s)=} & \eta c_{0, q}(0) \delta_{n, 0}-\frac{i q}{2}\left[\widetilde{c}_{n+1, q}(s)\right. \\
& \left.+2 n \widetilde{c}_{n-1, q}(s)\right]-\operatorname{in} \sigma\left[\widetilde{c}_{n-1, q+2}(s)\right. \\
& \left.-\widetilde{c}_{n-1, q-2}(s)\right]-2 \operatorname{in} \sigma h\left[\widetilde{c}_{n-1, q+1}(s)\right. \\
& \left.-\widetilde{c}_{n-1, q-1}(s)\right] .
\end{aligned}
$$

Here all other $c_{n, q}(0)=0$ for $n \geqslant 1$ because $\left\langle\cos \phi H_{n}(\dot{\phi})\right\rangle_{0}$ $=0$ for the equilibrium Maxwell-Boltzmann distribution. In order to solve Eq. (15), we introduce the column vectors

$$
\widetilde{\mathbf{C}}_{1}(s)=\left(\begin{array}{c}
\vdots \\
\widetilde{c}_{0,-2}(s) \\
\widetilde{c}_{0,-1}(s) \\
\widetilde{c}_{0,1}(s) \\
\widetilde{c}_{0,2}(s) \\
\vdots
\end{array}\right) \text { and } \widetilde{\mathbf{C}}_{n}(s)=\left(\begin{array}{c}
\vdots \\
\widetilde{c}_{n-1,-2}(s) \\
\widetilde{c}_{n-1,-1}(s) \\
\widetilde{c}_{n-1,0}(s) \\
\widetilde{c}_{n-1,1}(s) \\
\widetilde{c}_{n-1,2}(s) \\
\vdots
\end{array}\right)(n \geqslant 2)
$$

Now, Eq. (15) can be rearranged as the set of matrix threeterm recurrence equations,

$$
\begin{aligned}
& {\left[\eta s+\beta^{\prime}(n-1)\right] \widetilde{\mathbf{C}}_{n}(s)-\mathbf{Q}_{n}^{+} \widetilde{\mathbf{C}}_{n+1}(s)-\mathbf{Q}_{n}^{-} \widetilde{\mathbf{C}}_{n-1}(s)} \\
& \quad=\eta \delta_{n, 1} \mathbf{C}_{1}(0),
\end{aligned}
$$

where the column vector $\mathbf{C}_{1}(0)$ and the matrices $\mathbf{Q}_{n}^{+}$and $\mathbf{Q}_{n}^{-}$ are given in Appendix I. By invoking the general method for solving the matrix recurrence in Eq. (16), ${ }^{2}$ we have the exact solution for the spectrum $\widetilde{\mathbf{C}}_{1}(s)$ in terms of a matrixcontinued fraction, viz.,

$$
\widetilde{\mathbf{C}}_{1}(s)=\eta \boldsymbol{\Delta}_{1}(s) \mathbf{C}_{1}(0),
$$

where the matrix-continued fraction $\boldsymbol{\Delta}_{n}(s)$ is defined by the recurrence equation

$$
\boldsymbol{\Delta}_{n}(s)=\left\{\left[\eta s+\beta^{\prime}(n-1)\right] \mathbf{I}-\mathbf{Q}_{n}^{+} \boldsymbol{\Delta}_{n+1}(s) \mathbf{Q}_{n+1}^{-}\right\}^{-1}
$$

and $\mathbf{I}$ is the unit matrix.

Having determined $\widetilde{\mathbf{C}}_{1}(s)$, we can evaluate the spectrum

$$
\widetilde{C}(\omega)=\frac{\widetilde{c}_{0,-1}(i \omega)+\widetilde{c}_{0,1}(i \omega)}{c_{0,-1}(0)+c_{0,1}(0)}
$$

of the equilibrium correlation function $C(t)$, the longitudinal complex susceptibility $\chi(\omega)=\chi^{\prime}(\omega)-i \chi^{\prime \prime}(\omega)$, and the correlation time $\tau_{\|}$. By using matrix-continued fractions, one can also estimate the smallest nonvanishing eigenvalue $\lambda_{1}$ of the Fokker-Planck operator [that is $\lambda_{1}$ of the hierarchy of Eq. (14)], Eq. (5), from the secular equation ${ }^{1,2}$

$$
\operatorname{det}\left[\lambda_{1} \tau_{N} \mathbf{I}+\mathbf{Q}_{1}+\mathbf{Q}_{1}^{+} \boldsymbol{\Delta}_{2}\left(-\lambda_{1}\right) \mathbf{Q}_{2}^{-}\right]=0 .
$$

We remark that $\lambda_{1}$ can also be evaluated from the half-width of the spectrum $\widetilde{C}(\omega)$ or, equivalently, from the lowfrequency maximum of the dielectric loss spectra $\chi^{\prime \prime}(\omega)$. By utilizing general properties of Fourier transforms, we may also obtain simple asymptotic equations for $\chi(\omega)$ in the lowand high-frequency limits. We have

$$
\frac{\chi^{\prime \prime}(\omega)}{\chi^{\prime}(0)}=\omega \int_{0}^{\infty} C_{\|}(t) d t+\cdots=\omega \tau_{\|}
$$

for $\omega \rightarrow 0$ and

$$
\frac{\chi^{\prime \prime}(\omega)}{\chi^{\prime}(0)} \sim-\frac{\dot{C}_{\|}(0)}{\omega}+\frac{\dddot{C}_{\|}(0)}{\omega^{3}}+\cdots
$$

for $\omega \rightarrow \infty$. Here $\dot{C}_{\|}(0)=0$ and

$$
\begin{aligned}
\dddot{C}_{\|}(0) & =\frac{\dddot{c}_{0,-1}(0)+\dddot{c}_{0,1}(0)}{c_{0,-1}(0)+c_{0,1}(0)} \\
& =\frac{\beta^{\prime}}{2 \eta^{3}}\left[1-\sigma+2 \sigma h \frac{c_{0,2}(0)}{c_{0,1}(0)}+\sigma \frac{c_{0,3}(0)}{c_{0,1}(0)}\right] .
\end{aligned}
$$

We remark that for free Brownian rotation of plane rotators $(\sigma=0$ and $h=0), \lambda_{1}$ becomes in the VLD and very high damping (VHD) limits

$$
\lambda_{1} \rightarrow 1 / \eta(\sqrt{\pi}) \text { and } \lambda_{1} \rightarrow 1 /\left(2 \eta \beta^{\prime}\right),
$$

respectively. Equations (23) provide bounds for the purpose of testing the results of numerical calculations. Moreover, for $\sigma=0$ and $h=0$, the calculation shows that the matrixcontinued fraction algorithm yields the same results as the exact analytic solution for the free rotational diffusion obtained in Ref. 28, viz.,

$$
\begin{aligned}
\chi(\omega)= & \frac{\mu^{2} N_{0}}{2 k T}\left\{1-\frac{i \beta^{\prime} \eta \omega}{1+i \beta^{\prime} \eta \omega} M[1,1+(1\right. \\
& \left.\left.\left.+i \beta^{\prime} \eta \omega\right) /\left(2{\beta^{\prime 2}}^{2}\right), 1 /\left(2 \beta^{\prime 2}\right)\right]\right\},
\end{aligned}
$$

where $M(a, b, z)$ is the confluent hypergeometric (Kummer) function. ${ }^{27}$

\section{MEL'NIKOV-MESHKOV FORMULA FOR $\lambda_{1}$}

By solving the Fokker-Planck equation converted to an energy-action diffusion equation by the Wiener-Hopf method, Mel'nikov and Meshkov have evaluated the inverse 
of the longest relaxation time $\tau^{-1}=\lambda_{1}$ for the translational motion of a Brownian particle of mass $M$ in a double-well potential $V(x)$ with nonequivalent wells as ${ }^{12}$

$$
\tau=\frac{A\left[\beta\left(S_{1}+S_{2}\right) / k T\right]}{A\left(\beta S_{1} / k T\right) A\left(\beta S_{2} / k T\right)} \tau_{\text {IHD }}
$$

Here $\tau_{\text {IHD }}$ is the longest relaxation time in the IHD limit which can be estimated as

$$
\tau_{\mathrm{IHD}}^{-1}=\Gamma_{12}^{\mathrm{IHD}}+\Gamma_{21}^{\mathrm{IHD}},
$$

where $\Gamma_{12}^{\mathrm{IHD}}$ and $\Gamma_{21}^{\mathrm{IHD}}$ are the Kramers IHD escape rates from wells 1 and 2, respectively, $\beta$ is a damping coefficient for translational motion, $S_{1}$ and $S_{2}$ are the corresponding action variables, and

$$
A(d)=\exp \left[\frac{1}{\pi} \int_{0}^{\infty} \frac{\ln \left[1-\exp \left\{-d\left(\lambda^{2}+1 / 4\right)\right\}\right]}{\lambda^{2}+1 / 4} d \lambda\right]
$$

the function $A(d)$ involving the actions has the following properties: $^{12}$

$$
A(d) \rightarrow 1 \text { as } d \rightarrow \infty \text { and } A(d) / d \rightarrow 1 \text { as } d \rightarrow 0 .
$$

The leading factor on the right-hand side of Eq. (25) is the correction to the IHD escape rate due to Mel'nikov and Meshkov (the depopulation factor). The escape rates $\Gamma_{12}^{\mathrm{IHD}}$ and $\Gamma_{21}^{\mathrm{IHD}}$ are evaluated from the Kramers IHD formula ${ }^{8}$

$$
\Gamma_{i j} \sim \frac{\omega_{a}^{i}}{2 \pi}\left(\sqrt{1+\frac{\beta^{2}}{4 \omega_{c}^{i 2}}}-\frac{\beta}{2 \omega_{c}^{i}}\right) e^{-\Delta V_{i} / k T}
$$

where $\omega_{a}^{i}=\sqrt{V^{\prime \prime}\left(a_{i}\right) / M}$ and $\omega_{c}^{i}=\sqrt{\left|V^{\prime \prime}(c)\right| / M}$ are the angular frequencies of oscillation of a particle in the potential well $i$ at minimum $a_{i}$ and at the barrier coordinate $c$, the double prime denotes the second derivative with respect to $x$, and $\Delta V_{i}=V(c)-V\left(a_{i}\right)$ is the potential barrier.

The detailed derivation of the Melnikov and Meshkov formula for orientational relaxation of a planar rotator in two equivalent wells has been given in Ref. 23. This derivation can readily be generalized to the calculation of the longest relaxation time of the orientational relaxation in the potential with two nonequivalent wells, Eq. (3), yielding the universal formula in Eq. (25), where $\tau_{\mathrm{IHD}}^{-1}$ is now given by (after appropriate changes of notation)

$$
\begin{aligned}
& \tau_{\mathrm{IHD}}^{-1}=\frac{1}{\pi \eta}\left[\sqrt{\frac{\beta^{\prime 2}}{4}+\frac{\eta^{2}}{I}\left|V^{\prime \prime}\left(\phi_{0}\right)\right|}-\frac{\beta^{\prime}}{2}\right] \\
& \times\left[\sqrt{\frac{V^{\prime \prime}(0)}{\left|V^{\prime \prime}\left(\phi_{0}\right)\right|}} e^{\Delta V_{1} /(k T)}+\sqrt{\frac{V^{\prime \prime}(\pi)}{\left|V^{\prime \prime}\left(\phi_{0}\right)\right|}} e^{\Delta V_{2} /(k T)}\right] \\
& =\frac{e^{-2 \sigma(1-h)^{2}}}{2 \pi \eta}\left[\sqrt{\beta^{\prime 2}+8 \sigma\left(1-h^{2}\right)}-\beta^{\prime}\right] \\
& \times\left[\frac{1}{\sqrt{1-h}} e^{-8 \sigma h}+\frac{1}{\sqrt{1+h}}\right],
\end{aligned}
$$

where $\phi_{1}=0$ and $\phi_{2}=\pi$ are the potential minima in wells 1 and 2, $\phi_{0}=\pi-\arccos h$ is the barrier coordinate found from the condition $V\left(\phi_{0}\right)=0$ (see Fig. 1),

$$
\begin{aligned}
& \frac{\Delta V_{1,2}}{k T}=-2 \sigma(1 \pm h)^{2}, \quad \frac{\eta^{2}}{I}\left|V^{\prime \prime}\left(\phi_{0}\right)\right|=2 \sigma\left(1-h^{2}\right), \\
& \frac{\eta^{2}}{I} V^{\prime \prime}(0)=2 \sigma(1+h), \quad \frac{\eta^{2}}{I} V^{\prime \prime}(\pi)=2 \sigma(1-h), \\
& \frac{\beta S_{1}}{k T}=\frac{\beta}{k T} \int_{-\phi_{0}}^{\phi_{0}} \sqrt{-2 I V(\phi)} d \phi=4 \beta^{\prime} \sqrt{2 \sigma}\left(\sqrt{1-h^{2}}\right. \\
& \quad+h \pi-h \arccos h), \\
& \frac{\beta S_{2}}{k T}=\frac{\beta}{k T} \int_{\phi_{0}}^{2 \pi-\phi_{0}} \sqrt{-2 I V(\phi)} d \phi \\
& =4 \beta^{\prime} \sqrt{2 \sigma}\left(\sqrt{1-h^{2}}-h \arccos h\right) .
\end{aligned}
$$

If $\beta^{\prime} \rightarrow \infty$, we have from Eqs. (25), (28), and (29) the VHD formula

$$
\tau_{\mathrm{VHD}}=\frac{\pi \eta \beta^{\prime} e^{2 \sigma(1-h)^{2}}}{2 \sigma\left(\sqrt{1-h}+\sqrt{1+h} e^{-8 \sigma h}\right)} .
$$

In like manner, in the VLD limit, $\beta^{\prime} \rightarrow 0$, we obtain

$$
\tau_{\mathrm{VLD}}=\frac{\pi \eta e^{2 \sigma(1-h)^{2}}\left(\pi h / 2+\sqrt{1-h^{2}}-h \arccos h\right)}{4 \beta^{\prime} \sigma\left(\sqrt{1-h}+\sqrt{1+h} e^{-8 \sigma h}\right)\left(h \pi+\sqrt{1-h^{2}}-h \arccos h\right)\left(\sqrt{1-h^{2}}-h \arccos h\right)} .
$$

For $h=0$, the relaxation times from Eqs. (29)-(31) reduce to those obtained in Ref. 23 for equivalent wells.

\section{THE VHD AND VLD ASYMPTOTES FOR $\tau_{\|}$}

As far as the calculation of the longitudinal correlation time $\tau_{\|}$is concerned we remark that an accurate analytic estimation of $\tau_{\|}$from Eqs. (8) and (10) is in principle a much more complicated problem than the evaluation of the smallest nonvanishing eigenvalue alone since all the other eigen- values contribute to $\tau_{\|}$. Fortunately, an accurate method of the estimation $\tau_{\|}$in the VHD and VLD limits exists. This method is based on a mean first passage time calculation first suggested by $\mathrm{Szabo}^{29}$ in the context of a theory of polarized fluorescent emission in uniaxial liquid crystals based on the Smoluchowski equation. However, it may be used for all systems with dynamics governed by single-variable FokkerPlanck equations to yield the correlation time in terms of the equilibrium (stationary) distribution function $W_{0}$ and diffu- 
sion coefficient $D^{(2)}$ only (see, e.g., Ref. 1, Sec. S.9 or Ref. 2, Sec. 2.10). The advantage of such a method is that it allows us to obtain for the problem under consideration the VHD and VLD asymptotes, valid for all barrier heights including very low barriers, where asymptotic methods (like that of Melnikov-Meshkov) are not applicable.

In the high damping limit $\left(\beta^{\prime} \gg 1\right)$ the appropriate single-variable Fokker-Planck (Smoluchowski) equation for the probability density function $W(\phi, t)$ of the orientations of rotators is ${ }^{2,30}$

$$
\begin{aligned}
\frac{\partial}{\partial t} W(\phi, t)= & \frac{\sigma}{\beta^{\prime} \eta} \frac{\partial}{\partial \phi}[(\sin 2 \phi+2 h \sin \phi) W(\phi, t)] \\
& +\frac{1}{2 \beta^{\prime} \eta} \frac{\partial^{2}}{\partial \phi^{2}} W(\phi, t) .
\end{aligned}
$$

Thus, noting that here the diffusion coefficient $D^{(2)}$ $=\left(2 \beta^{\prime} \eta\right)^{-1}$, the correlation time of the longitudinal dipole moment autocorrelation function in the VHD limit is given by

$$
\begin{aligned}
\tau_{\|} \sim & \tau_{\|}^{\mathrm{VHD}}=\frac{2 \beta^{\prime} \eta}{\left\langle\cos ^{2} \phi\right\rangle_{0}-\langle\cos \phi\rangle_{0}^{2}} \int_{0}^{2 \pi} \frac{1}{W_{0}(\phi)} \\
& \times\left[\int_{0}^{\phi}\left(\cos \phi^{\prime}-\left\langle\cos \phi^{\prime}\right\rangle_{0}\right) W_{0}\left(\phi^{\prime}\right) d \phi^{\prime}\right]^{2} d \phi,
\end{aligned}
$$

where $W_{0}(\phi)$ is the equilibrium Boltzmann distribution function given by

$$
\begin{aligned}
W_{0}(\phi) & =\int W_{0}(\dot{\phi}, \phi) d \dot{\phi} \\
& =e^{2 \sigma \cos ^{2} \phi+4 \sigma h \cos \phi} / \int_{0}^{2 \pi} e^{2 \sigma \cos ^{2} \phi+4 \sigma h \cos \phi} d \phi
\end{aligned}
$$

[this function is a stationary solution of Eq. (32)]; $\left\langle\cos ^{2} \phi\right\rangle_{0}$ and $\langle\cos \phi\rangle_{0}$ can be calculated as described in Appendix I.

In the opposite low damping limit $\left(\beta^{\prime} \ll 1\right)$, in order to obtain a single-variable Fokker-Planck equation, one may introduce as variables the energy of the dipole

$$
\varepsilon=\eta^{2} \dot{\phi}^{2}-2 \sigma \cos ^{2} \phi-4 \sigma h \cos \phi
$$

and the time $w$ (phase) measured along a closed trajectory in phase space as action-angle variables. ${ }^{31}$ The energy $\varepsilon$ varies very slowly with time. Consequently, it is a slow variable in comparison to the phase $w$. By averaging the Fokker-Planck equation (4) over the fast phase variable $w$, Praestgaard and van Kampen ${ }^{31}$ derived a single-variable Fokker-Planck equation for the probability density function $W(\varepsilon, t)$ in energy space (in our notation),

$$
\begin{aligned}
\frac{\partial}{\partial t} W(\varepsilon, t)= & \frac{2 \beta^{\prime}}{\eta}\left[\frac{\partial}{\partial \varepsilon}\left(\eta^{2} \overline{\overline{\dot{\phi}^{2}}}(\varepsilon)-\frac{1}{2}\right)\right. \\
& \left.+\eta^{2} \frac{\partial^{2}}{\partial \varepsilon^{2}} \overline{\overline{\dot{\phi}^{2}}}(\varepsilon)\right] W(\varepsilon, t),
\end{aligned}
$$

where the double overbar denotes averaging over the fast phase variable. Noting that the diffusion coefficient $D^{(2)}$ $=2 \beta^{\prime} \eta \overline{\overline{\dot{\phi}^{2}}}(\varepsilon)$, the correlation time $\tau_{\|}$is then given by

$$
\tau_{\|} \sim \tau_{\|}^{\mathrm{VLD}}=\eta \sqrt{\pi}+\tau_{+}+\tau_{-},
$$

where

$$
\tau_{ \pm}=\frac{1}{\left(\left\langle\cos ^{2} \phi\right\rangle_{0}-\langle\cos \phi\rangle_{0}^{2}\right)} \int_{-2 \sigma(1 \pm 2 h)}^{\infty} \frac{\left.\left(\int_{-2 \sigma(1 \pm 2 h)}^{\varepsilon} \overline{(\overline{\cos \phi}}\left(\varepsilon^{\prime}\right)-\langle\cos \phi\rangle_{0}\right) W_{0}\left(\varepsilon^{\prime}\right) d \varepsilon^{\prime}\right)^{2}}{2 \beta^{\prime} \eta \overline{\overline{\dot{\phi}^{2}}}(\varepsilon) W_{0}(\varepsilon)} d \varepsilon
$$

and $\eta^{2} \overline{\overline{\dot{\phi}^{2}}}(\varepsilon)=\varepsilon+2 \sigma \overline{\overline{\cos \phi^{2}}}(\varepsilon)+4 \sigma h \overline{\overline{\cos \phi}}(\varepsilon)$. The calculation of $W_{0}(\varepsilon), \overline{\overline{\cos \phi^{2}}}(\varepsilon), \overline{\overline{\cos \phi}}(\varepsilon)$, and the integrals in Eqs. (38) is described in Appendix II. The term $\eta \sqrt{\pi}$ in Eq. (37) represents the contribution of the free rotation to the correlation time (it is independent of $\beta^{\prime}$ and may be obtained from the solution of the undamped equation $\dot{W}=0$ ).

The regions of applicability of the asymptotes from Eqs. (33) and (37) are the same as for the corresponding FokkerPlanck equations (32) and (36), viz., the VHD $\left(\beta^{\prime} \gg 1\right)$ and VLD $\left(\beta^{\prime} \ll 1\right)$ regions, respectively; in practice, Eqs. (33) and (37) may be used for $\beta^{\prime}>5$ and $\beta^{\prime}<0.01$.

\section{RESULTS AND DISCUSSION}

The imaginary $\chi^{\prime \prime}(\omega)$ part of the complex susceptibility for various values of the anisotropy parameter $\sigma$, the asymmetry parameter $h$, and the friction coefficient $\beta^{\prime}$ are shown in Fig. 2 [the calculations were carried out for $\mu^{2} N_{0} /(k T)$ $=1]$. In general, three bands appear in the dielectric loss $\chi^{\prime \prime}(\omega)$ spectra. One relaxation band dominates the lowfrequency part of the spectra and is due to the slow overbarrier relaxation of the dipoles in the double-well potential. The characteristic frequency $\omega_{R} \approx \lambda_{1}$ of this low-frequency band strongly depends on $\sigma$ and $h$ as well as on the friction parameter $\beta^{\prime}$. Regarding the barrier height or $\sigma$ dependence, 

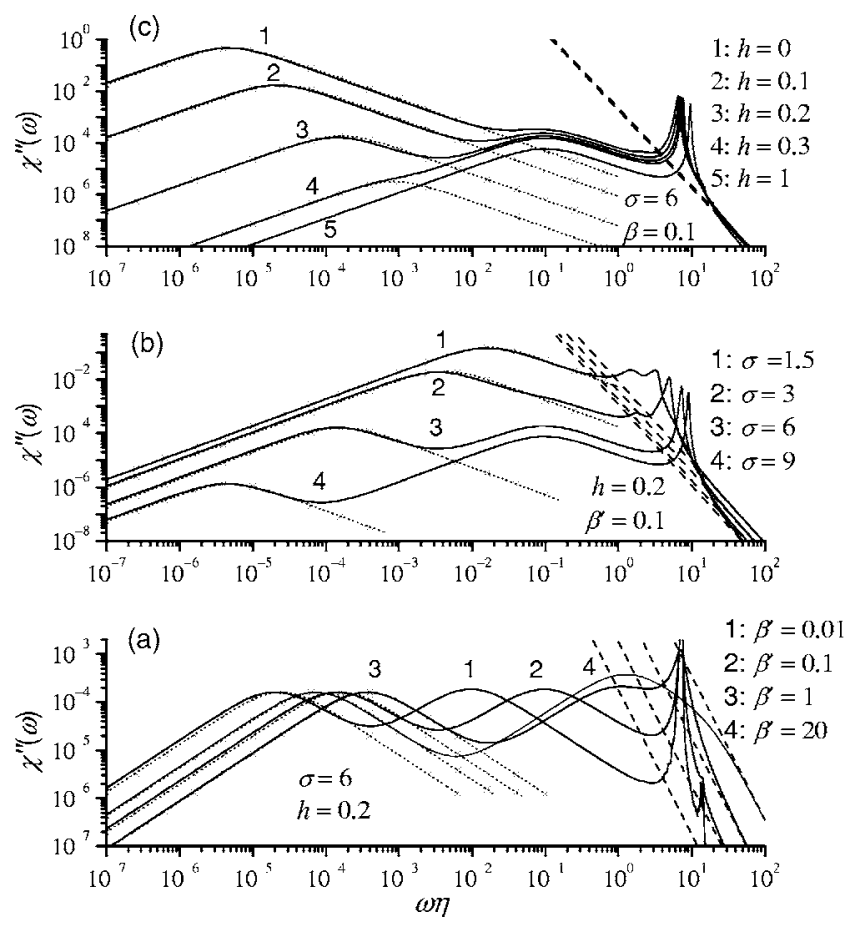

FIG. 2. Dielectric loss spectra (solid lines) for various values of $\sigma, \beta$, and $h$. The Debye spectra [Eq. (39)] are shown by the dotted lines with crosses. The high-frequency asymptotes [Eq. (21)] are shown by the dashed lines.

the frequency $\omega_{R}$ decreases exponentially as $\sigma$ is raised. This behavior occurs because the probability of escape of a dipole from one well to another over the potential barrier exponentially decreases with increasing $\sigma$. As far as the dependence of the low-frequency part of the spectrum for large friction (small inertial effects) $\beta^{\prime}>10$ is concerned, the frequency $\omega_{R}$ decreases as $\beta^{\prime}$ increases as is apparent by inspection of curves in Fig. 2(c). For small friction (large inertial effects) $\beta^{\prime}<0.1$ the frequency $\omega_{R}$ decreases with decreasing $\beta^{\prime}$ for given values of $\sigma$ and $h$ [cf. curves 1-3 in Fig. 2(c)]. This low-frequency part of the spectrum may by approximated by the Debye equation

$$
\frac{\chi(\omega)}{\chi^{\prime}(0)}=\frac{1-\Delta}{1+i \omega \tau}+\Delta
$$

where the longest relaxation time $\tau=1 / \lambda_{1}$ is given by the universal equation (25) and $\Delta$ is a parameter accounting for the contribution of the high-frequency modes. As $h$ increases, the magnitude of the low-frequency band decreases and this band disappears entirely for $h>1$ [see Fig. 2(a)] where the double-well nature of the potential is destroyed.

A very high-frequency band is visible in all the figures due to the fast inertial librations of the dipoles in the potential wells. This band corresponds to the terahertz (farinfrared) range of frequencies and is usually associated with the Poley absorption. ${ }^{32}$ For $\sigma \gg 1$ and $h \approx 0$, the characteristic frequency of librations $\omega_{L}$ increases as $\sim \eta^{-1} \sqrt{\sigma}$. As far as the behavior as a function of $\beta^{\prime}$ is concerned, the amplitude of the high-frequency band decreases progressively with increasing $\beta^{\prime}$, as one would intuitively expect. On the other hand, for small friction (large inertial effects) $\beta^{\prime} \ll 1$, a fine structure appears in the high-frequency part of the spectra [due to resonances at high harmonic frequencies of the almost free motion in the (anharmonic) potential]. We remark that the high-frequency $\left(\omega \gg \omega_{L}\right)$ behavior of $\hat{\chi}^{\prime \prime}(\omega)$ is entirely determined by the inertia of the system and is described by Eq. (21) (these high-frequency asymptotes are also shown in Fig. 2 for comparison). Finally, as we have mentioned in the Introduction it is apparent that between the low-frequency and very high-frequency bands, a third band exists in the dielectric loss spectra. This band is due to the high-frequency relaxation or decay modes of the dipoles in the potential wells which will always exist in the spectra even in the noninertial limit. ${ }^{2}$ Such relaxation modes are generally termed the intrawell modes.

Thus one may conclude that the asymmetric double-well potential gives rise to three distinguished relaxation processes: (i) a slow low-frequency overbarrier mode, (ii) relatively fast intermediate frequency modes due to the near degenerate exponential decays in the wells of the potential, and (iii) fast high-frequency oscillatory (librational) modes in the wells of the potential. If the asymmetry parameter which is strongly dependent on the precise details of the potential ${ }^{2}$ is regarded as a structural relaxation parameter in the sense used by Gilroy and Philips ${ }^{33}$ it appears that the structural parameter can effectively destroy the low-frequency relaxation mode due to the overbarrier relaxation so that above the critical value of that parameter the system is no longer effectively a multiwell system as the population behaves like that of a single-well potential. Hence we only have fast near degenerate exponential decays in the well accompanied by high-frequency oscillatory modes arising from inertial effects due to the small oscillations in the well [c.f. Fig. 2(a)].

The greatest relaxation time $\tau$ predicted by the Mel'nikov and Meshkov ${ }^{12}$ method [Eq. (25)] and the smallest nonvanishing eigenvalue $\lambda_{1}$ calculated numerically by matrix-continued fraction methods as the low-frequency maximum of the dielectric loss spectra $\chi^{\prime \prime}(\omega)$ are shown in Fig. 3 as functions of $\beta^{\prime}$ for various values of $h$ and $\sigma$. Here, the VHD [Eq. (30)], IHD [Eq. (29)], and VLD [Eq. (31)] asymptotes are also shown for comparison. Apparently in the high barrier limit, Eq. (25) provides a good approximation to $\lambda_{1}$ for all $\beta^{\prime}$ including the VHD, VLD, and turnover regions. Furthermore, Eq. (25) yields a reasonable estimate for $\lambda_{1}$ even for $\sigma \approx 1$.

The quantitative agreement in damping behavior may be explained as follows. The behavior of the escape rate as a function of the barrier height parameter $\sigma$ for large $\sigma$ is approximately an Arrhenius type and arises from an equilibrium property of the system (namely, the Boltzmann distribution at the bottom of the well). On the other hand, the damping dependence of the escape rate is due to nonequilibrium (dynamical) properties of the system and so is contained in the prefactor $A$ only, the detailed nature of which depends on the behavior of the energy distribution function at the barrier points. ${ }^{10}$ The Mel'nikov-Meshkov approach ${ }^{11}$ yields the distribution function at the saddle point for all values of the damping allowing one to evaluate the damping dependence of the prefactor $A$ in Eq. (1). We remark that as emphasized by Kramers, it is hardly ever of any practical importance to improve on the accuracy of the IHD or VLD 

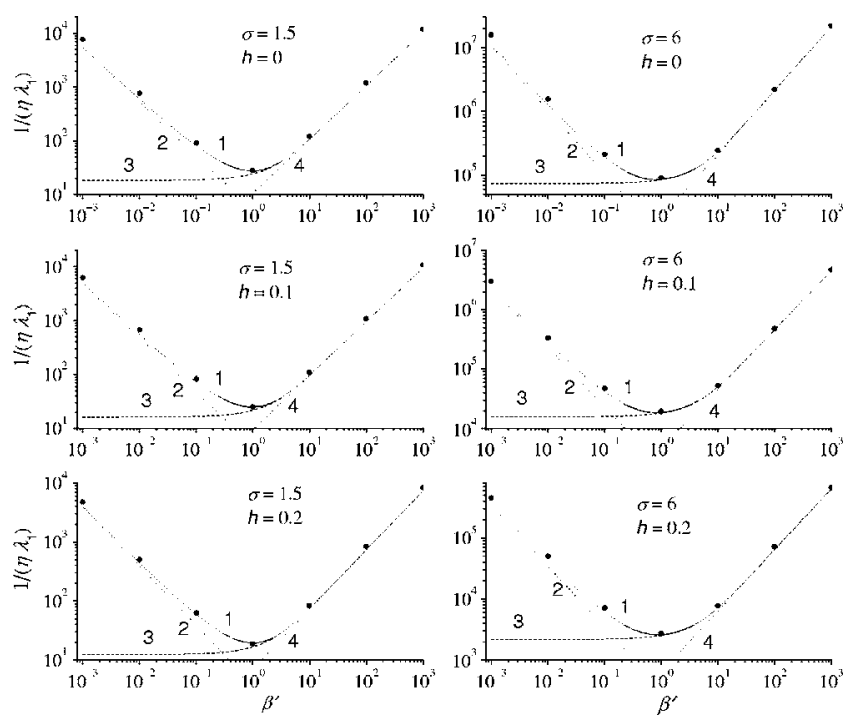

FIG. 3. Longest relaxation time $\tau=1 / \lambda_{1}$ vs $\beta^{\prime}$ for $\sigma=1.5$ and 6 , and $h$ $=0.0,0.1$ and 0.2 . solid lines 1 : the universal equation (25); dot-dashed lines 4: the VHD equation (30); dashed lines line 3: the IHD equation (29); dotted 4: the VLD equation (31); and filled circles: exact matrix-continued fraction solution.

formulas themselves because in experimental situations where relaxation is studied, one has only estimates of the prefactor within a certain degree of accuracy which is difficult to evaluate. For example, little detailed information about the value of $\beta^{\prime}$ exists. Nevertheless, it is important to predict the behavior of the relaxation times as a function of $\beta^{\prime}$ using analytical methods such as the one used in this paper because of the detailed information such methods yield about the mechanisms underlying the relaxation process. The description of the relaxation processes in the context of Eq. (25) neglects quantum effects. These effects are important at very low temperatures and necessitate an appropriate quantum-mechanical treatment. Mel'nikov and Mesnkov ${ }^{12}$ and Rips and Pollak ${ }^{15}$ have extended the bridging integral method for mechanical particles to account for quantum effects in a semiclassical way.

These VHD and VLD asymptotes for $\tau_{\|}$are shown in Figs. 4 and 5. In Fig. 4, we compare Eqs. (33) and (37) with the exact numerical solution for the correlation time $\tau_{\|}$and the Mel'nikov-Meshkov universal formula. Here we see the asymmetry effect alluded to in the Introduction where for a certain value of $h$, namely, $h_{c} \approx 0.18$, the Arrhenius behavior (exponential increase with increasing barrier height) of the correlation time $\tau_{\|}$disappears. Such an effect occurs at a critical value $h_{c}$ of the ratio $h$, i.e., bias field parameter/ anisotropy barrier height parameter, far less than the nucleation field which is the value needed to destroy the bistable nature of the potential. Thus in the low-temperature limit, the overall relaxation process is no longer dominated by the slow decay or interwell mode associated with the barrier crossing at values of $h$ in excess of the critical value. The phenomenon was first discovered by (by numerical methods) Coffey et al. $^{4}$ and later explained by Garanin ${ }^{5}$ for the very similar problem of the magnetization relaxation of a uniaxial superparamagnetic particle subjected to a dc magnetic field. Garanin $^{5}$ showed that this effect is a natural consequence of
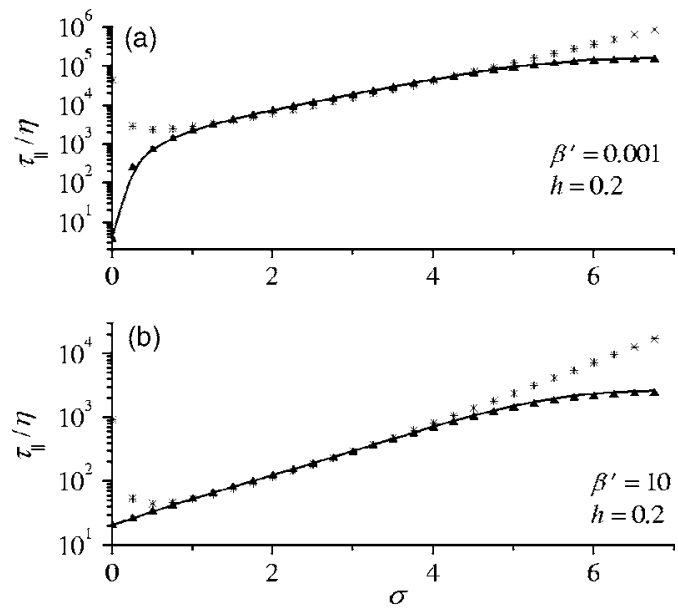

FIG. 4. $\tau_{\|} / \eta$ vs $\sigma$ for $\beta^{\prime}=0.001$ [(a) very low damping $]$ and $\beta^{\prime}=10[$ [(b) high damping]. Solid lines: exact matrix-continued fraction solution for the correlation time $\tau_{\|}$; stars: the universal Mel'nikov-Meshkov equation (25); and triangles: Eqs. (33) and (37) for the VHD and VLD regions, respectively.

the population depletion of the shallower of the two potential wells (which are involved in the barrier crossing) by the uniform field. Essentially when a significant part of the population of the shallow well has descended to the deeper well the enhanced population in that well cannot escape because of the high potential barrier which it has to overcome. Further, in Ref. 34 it has been shown that this depletion effect always exists in relaxation in bistable potentials and it has also been asserted ${ }^{2}$ that such an effect is a general feature of relaxation in biased double-well potentials. In the particular application to the present problem, the long-time behavior of $C(t)$ at low temperatures $(\sigma, \xi \gg 1)$ may be approximated by two exponentials corresponding to overbarrier and intrawell relaxation processes (on neglecting the contribution of librational modes),

$$
C(t) \approx(1-\Delta) e^{-t \lambda_{1}}+\Delta e^{-t / \tau_{\text {well }}}
$$

where $\tau_{\text {well }}$ is the effective relaxation time in the deep well and so has a weak temperature dependence. According to Eqs. (10) and (40),
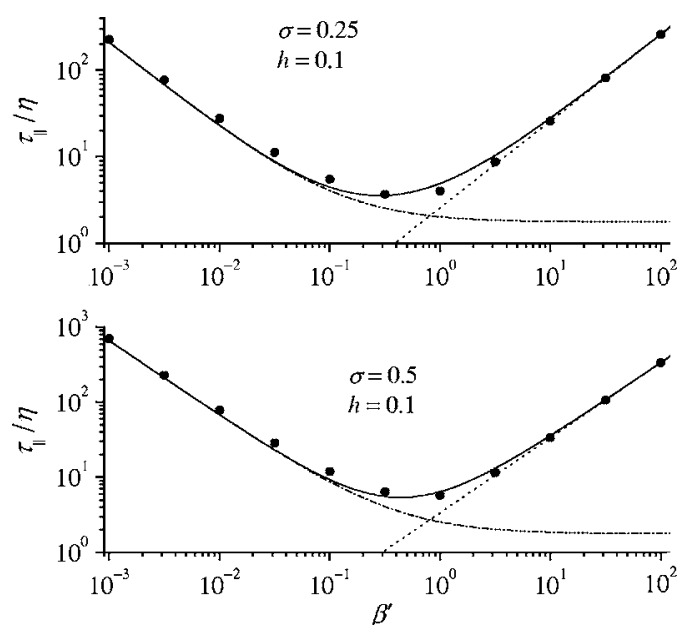

FIG. 5. $\tau_{\|} / \eta$ vs $\beta^{\prime}$ for small barrier heights $\sigma=0.25$ and 0.5 and $h=0.1$. Filled circles: exact matrix-continued fraction solution for the correlation time $\tau_{\|}$; dashed-dotted lines 2: the VLD equation (37); dotted lines 3: the VHD equation (33); and solid lines 1: Eq. (42). 


$$
\tau_{\|} \approx(1-\Delta) \lambda_{1}^{-1}+\Delta \tau_{\text {well }}
$$

If $h<h_{c}$, the quantity $(1-\Delta) \lambda_{1}^{-1}$ increases exponentially as the temperature $T$ decreases and so $(1-\Delta) \lambda_{1}^{-1}$ determines completely the temperature dependence of the correlation time $\tau_{\|}$. If $h>h_{c}$, on the other hand, the quantity $(1-\Delta) \lambda_{1}^{-1}$ decreases exponentially as $T$ decreases [due to the depletion effect $(1-\Delta) \lambda_{1}^{-1}$ is exponentially small in spite of the fact that $\lambda_{1}^{-1}$ is exponentially large], thus $\tau_{\|}$no longer has Arrhenius behavior and now differs exponentially from $\lambda_{1}^{-1}$. Thus for $h=h_{c}$, the relaxation switches from being dominated by the behavior of the longest-lived relaxation mode associated with $\lambda_{1}^{-1}$ that is the inverse Kramers escape rate to being dominated by the fast relaxation processes in the deep well of the potential because of the depletion of the upper (shallow) well at low temperatures. 5,34

In Fig. 5, we compare the VHD and VLD correlation time equations (33) and (37) with the exact numerical solution for the correlation time at small barriers. Here a simple ad hoc extrapolating equation ${ }^{9}$

$$
\tau_{\|} \sim \tau_{\|}^{\mathrm{VLD}}+\tau_{\|}^{\mathrm{VHD}}
$$

provides a satisfactory estimate of the correlation time $\tau_{\|}$for all dampings and also the greatest relaxation time provided $h<h_{c}$ because in that situation $\tau_{\|} \approx \lambda_{1}^{-1}$. We emphasize that Eqs. (33), (37), and (42) can be used for all barrier heights $\sigma$, where the Melnikov-Meshkov universal formula is not applicable.

Thus the simple analytic Mel'nikov-Meshkov formula, ${ }^{11,12} \mathrm{Eq}$. (25), for the longest relaxation time bridging the VLD and IHD escape rates as a function of the dissipation parameter $\beta^{\prime}$ yields satisfactory agreement with the numerical results for fixed axis rotators in an asymmetric double-well potential for all values of $\beta^{\prime}$. Equation (25) allows one also to estimate accurately the damping dependence of the low-frequency part of the spectra of the equilibrium correlation function $C(t)$ and of the longitudinal complex susceptibility $\chi(\omega)$. Moreover it allows one to estimate the contribution of the overbarrier relaxation mode to the correlation time $\tau_{\|}$.

\section{ACKNOWLEDGMENT}

One of the authors (S.V.T.) would like to thank the Trinity College Dublin Trust for financial support for this work.

\section{APPENDIX A: EQUATIONS FOR $\mathrm{C}_{1}(0), \mathrm{Q}_{n}^{+}$, AND $Q_{n}^{-}$}

The column vector $\mathbf{C}_{1}(0)$ and the matrices $\mathbf{Q}_{n}^{+}$and $\mathbf{Q}_{n}^{-}$ are given by

$$
\begin{aligned}
& \mathbf{C}_{1}(0)=\left(\begin{array}{c}
\vdots \\
c_{0,-2}(0) \\
c_{0,-1}(0) \\
c_{0,1}(0) \\
c_{0,2}(0) \\
\vdots
\end{array}\right), \\
& \mathbf{Q}_{n}^{+}=-\frac{i}{2}\left(\begin{array}{ccccccc}
\ddots & \vdots & \vdots & \vdots & \vdots & \vdots & . \\
\cdots & -2 & 0 & 0 & 0 & 0 & \cdots \\
\cdots & 0 & -1 & 0 & 0 & 0 & \cdots \\
\cdots & 0 & 0 & 0 & 0 & 0 & \cdots \\
\cdots & 0 & 0 & 0 & 1 & 0 & \cdots \\
\cdots & 0 & 0 & 0 & 0 & 2 & \cdots \\
. & \vdots & \vdots & \vdots & \vdots & \vdots & \ddots
\end{array}\right) \text {, }
\end{aligned}
$$

$$
\mathbf{Q}_{n}^{-}=-i(n-1)\left(\begin{array}{ccccccccccc}
\ddots & \vdots & \vdots & \vdots & \vdots & \vdots & \vdots & \vdots & \vdots & \vdots & . \\
\cdots & -\sigma & -2 \sigma h & -2 & 2 \sigma h & \sigma & 0 & 0 & 0 & 0 & \cdots \\
\cdots & 0 & -\sigma & -2 \sigma h & -1 & 2 \sigma h & \sigma & 0 & 0 & 0 & \cdots \\
\cdots & 0 & 0 & -\sigma & -2 \sigma h & 0 & 2 \sigma h & \sigma & 0 & 0 & \cdots \\
\cdots & 0 & 0 & 0 & -\sigma & -2 \sigma h & 1 & 2 \sigma h & \sigma & 0 & \cdots \\
\cdots & 0 & 0 & 0 & 0 & -\sigma & -2 \sigma h & 2 & 2 \sigma h & \sigma & \cdots \\
. & \vdots & \vdots & \vdots & \vdots & \vdots & \vdots & \vdots & \vdots & \vdots & \ddots
\end{array}\right)
$$

The exceptions are the matrices $\mathbf{Q}_{1}^{+}$and $\mathbf{Q}_{2}^{-}$, which are given by

$$
\mathbf{Q}_{1}^{+}=-\frac{i}{2}\left(\begin{array}{ccccccc}
\ddots & \vdots & \vdots & \vdots & \vdots & \vdots & . \\
\cdots & -2 & 0 & 0 & 0 & 0 & \cdots \\
\cdots & 0 & -1 & 0 & 0 & 0 & \cdots \\
\cdots & 0 & 0 & 0 & 1 & 0 & \cdots \\
\cdots & 0 & 0 & 0 & 0 & 2 & \cdots \\
. & \vdots & \vdots & \vdots & \vdots & \vdots & \ddots
\end{array}\right)
$$




$$
\mathbf{Q}_{2}^{-}=-i\left(\begin{array}{cccccccccc}
\ddots & \vdots & \vdots & \vdots & \vdots & \vdots & \vdots & \vdots & \vdots & . \\
\cdots & -\sigma & -2 \sigma h & -2 & 2 \sigma h & 0 & 0 & 0 & 0 & \cdots \\
\cdots & 0 & -\sigma & -2 \sigma h & -1 & \sigma & 0 & 0 & 0 & \cdots \\
\cdots & 0 & 0 & -\sigma & -2 \sigma h & 2 \sigma h & \sigma & 0 & 0 & \cdots \\
\cdots & 0 & 0 & 0 & -\sigma & 1 & 2 \sigma h & \sigma & 0 & \cdots \\
\cdots & 0 & 0 & 0 & 0 & -2 \sigma h & 2 & 2 \sigma h & \sigma & \cdots \\
. & \vdots & \vdots & \vdots & \vdots & \vdots & \vdots & \vdots & \vdots & \ddots
\end{array}\right) .
$$

The initial conditions $c_{0, q}(0)$,

$$
\begin{aligned}
c_{0, q}(0)= & \left\langle e^{-i q \phi} \cos \phi\right\rangle_{0}-\langle\cos \phi\rangle_{0}\left\langle e^{-i q \phi}\right\rangle_{0} \\
= & \int_{0}^{2 \pi} \cos \phi e^{-i q \phi} W_{0}(\phi) d \phi \\
& -\int_{0}^{2 \pi} \cos \phi W_{0}(\phi) d \phi \int_{0}^{2 \pi} e^{-i q \phi} W_{0}(\phi) d \phi,
\end{aligned}
$$

where $W_{0}(\phi)$ is the equilibrium distribution in configuration space defined by Eq. (34), can be evaluated analytically in terms of the modified Bessel functions of the first kind $I_{n}$ (Ref. 27) by noting that

$$
\begin{gathered}
\left\langle e^{-i q \phi} \cos \phi\right\rangle_{0}=\frac{\sum_{m=-\infty}^{\infty} I_{m}(\sigma)\left[I_{-2 m+q+1}(4 \sigma h)+I_{-2 m+q-1}(4 \sigma h)\right]}{2 \sum_{m=-\infty}^{\infty} I_{m}(\sigma) I_{-2 m}(4 \sigma h)}, \\
\left\langle e^{-i q \phi}\right\rangle_{0}=\frac{\sum_{m=-\infty}^{\infty} I_{m}(\sigma) I_{-2 m+q}(4 \sigma h)}{\sum_{m=-\infty}^{\infty} I_{m}(\sigma) I_{-2 m}(4 \sigma h)},
\end{gathered}
$$

and

$$
\langle\cos \phi\rangle_{0}=\frac{\sum_{m=-\infty}^{\infty} I_{m}(\sigma)\left[I_{-2 m+1}(4 \sigma h)+I_{-2 m-1}(4 \sigma h)\right]}{2 \sum_{m=-\infty}^{\infty} I_{m}(\sigma) I_{-2 m}(4 \sigma h)} .
$$

\section{APPENDIX B: EVALUATION OF AVERAGES IN THE UNDAMPED LIMIT}

In the very low damping limit $\left(\beta^{\prime} \ll 1\right)$, the energy of the dipole is not conserved but will vary very slowly with time (quasistationarity). Thus the dynamics of the system are described by the one-dimensional Fokker-Planck equation (36) and differ but little from those of the undamped limit $\left(\beta^{\prime}\right.$ $=0$ ). In the undamped limit, the energy $\varepsilon$ is a constant of the motion. Thus the dynamics of the dipole are described by the deterministic differential equation

$$
\left(\eta \frac{d \phi}{d t}\right)^{2}=\varepsilon+4 \sigma h \cos \phi+2 \sigma \cos ^{2} \phi
$$

or, by introducing a new variable $Z=\cos \phi$,

$$
\left(\eta \frac{d Z}{d t}\right)^{2}=2 \sigma\left(Z^{2}+2 h Z+\varepsilon^{\prime}\right)\left(1-Z^{2}\right)
$$

where $\varepsilon^{\prime}=\varepsilon /(2 \sigma)$. Equation (B1) has a solution ${ }^{35,36}$ in terms of the Jacobian doubly periodic elliptic functions $\operatorname{sn}(u \mid m)$ and $\operatorname{cn}(u \mid m):^{27}$

$$
\cos \phi(t)= \begin{cases} \pm \frac{1-a^{ \pm} \operatorname{sn}^{2}\left(M_{1} t / \eta+w^{ \pm}, m_{1}\right)}{1+a^{ \pm} \operatorname{sn}^{2}\left(M_{1} t / \eta+w^{ \pm}, m_{1}\right)}, & -1 \mp 2 h \leqslant \varepsilon^{\prime} \leqslant h^{2} \\ -\frac{a-\operatorname{cn}\left(M_{2} t / \eta+w, m_{2}\right)}{1-a \operatorname{cn}\left(M_{2} t / \eta+w, m_{2}\right)}, & \varepsilon^{\prime} \geqslant h^{2},\end{cases}
$$

where

$$
\begin{aligned}
& m_{1}=\frac{1-\varepsilon^{\prime}-2 \sqrt{h^{2}-\varepsilon^{\prime}}}{1-\varepsilon^{\prime}+2 \sqrt{h^{2}-\varepsilon^{\prime}}}, \quad m_{2}=\frac{1}{2}\left(1+\frac{1-\varepsilon^{\prime}}{\sqrt{\left(1+\varepsilon^{\prime}\right)^{2}-4 h^{2}}}\right), \\
& M_{1}=\sqrt{\sigma\left(1-\varepsilon^{\prime}+2 \sqrt{\left.h^{2}-\varepsilon^{\prime}\right) / 2}\right.}, \quad M_{2}=\sqrt{2 \sigma \sqrt{\left(1+\varepsilon^{\prime}\right)^{2}-4 h^{2}}}, \\
& a^{ \pm}=\frac{1 \pm h-\sqrt{h^{2}-\varepsilon^{\prime}}}{1 \mp h+\sqrt{h^{2}-\varepsilon^{\prime}}}, \quad a=\frac{2 h}{1+\varepsilon^{\prime}+\sqrt{\left(1+\varepsilon^{\prime}\right)^{2}-4 h^{2}}}, \\
& w^{ \pm}=\int_{0}^{\sqrt{[1 \mp \cos \phi(0)]]\left[a^{ \pm}(1 \pm \cos \phi(0))\right]}} \frac{d t}{\sqrt{\left(1-t^{2}\right)\left(1-m_{1} t^{2}\right)}}, \\
& w=\int_{0}^{\left.\left[\sin \phi(0) \sqrt{1-a^{2}}\right]\right][1+a \cos \phi(0)]} \frac{d t}{\sqrt{\left(1-t^{2}\right)\left(1-m_{2} t^{2}\right)}} .
\end{aligned}
$$

We remark that the three solutions given by Eq. (B2) correspond to the three possible domains of energy variations, viz., the oscillations in the deeper well (domain I: $-1-2 h$ $\leqslant \varepsilon^{\prime} \leqslant h^{2}$ ), the oscillations in the shallow well (domain II: $-1+2 h \leqslant \varepsilon^{\prime} \leqslant h^{2}$ ), and rotation (domain III: $h^{2} \leqslant \varepsilon^{\prime}<\infty$ ). The condition for the existence of two wells is $h<1$.

The function $\cos \phi(t)$ is a periodic function of its arguments $M_{1} t / \eta+w^{ \pm}$and $M_{2} t / \eta+w$ with the period $T$ given by $2 K\left(m_{1}\right)$ for $-1 \mp 2 h \leqslant \varepsilon^{\prime} \leqslant h^{2}$ and $4 K\left(m_{2}\right)$ for $\varepsilon^{\prime} \geqslant h^{2}$, where $K(m)$ is the complete elliptic integral of the first kind. Hence $\cos \phi(t)$ can be expanded in the Fourier series 


$$
\cos \phi\left(t^{\prime}\right)=\frac{1}{2} c_{0}+\sum_{n=1}^{\infty} c_{n} \cos \left(\frac{2 \pi n\left(t^{\prime}+w\right)}{T}\right)
$$

with coefficients

$$
c_{n}=\frac{2}{T} \int_{-T / 2}^{T / 2} \cos \phi\left(t^{\prime}\right) e^{-2 i \pi n t^{\prime} / T} d t^{\prime} .
$$

Thus, we can readily obtain $\cos \phi$ averaged over the phase $w$ from Eq. (B3), viz.,

$$
\overline{\overline{\cos \phi(\varepsilon, w)}}=\frac{1}{T} \int_{-T / 2}^{T / 2} \cos \phi(\varepsilon, w) d w=\frac{1}{2} c_{0} .
$$

The coefficients $c_{0}$ can be calculated analytically using table integrals from Ref. 37. We have

$$
\begin{aligned}
& \overline{\overline{\cos \phi}}(\varepsilon) \\
& = \begin{cases}\mp 1 \pm 2 \frac{\Pi\left(-a^{ \pm}, m_{1}\right)}{K\left(m_{1}\right)}, & -1 \mp 2 h \leqslant \varepsilon^{\prime} \leqslant h^{2} \\
-\frac{1}{a}+\frac{\left(1-a^{2}\right)}{a K\left(m_{2}\right) \sqrt{1-m_{2}}} \Pi\left(a^{2}, \frac{m_{2}}{m_{2}-1}\right), & \varepsilon^{\prime} \geqslant h^{2}\end{cases}
\end{aligned}
$$

and

$$
\begin{aligned}
& \overline{\overline{\cos ^{2} \phi}}(\varepsilon) \\
& = \begin{cases}\frac{a^{ \pm}-1}{a^{ \pm}+1}+2 \frac{a^{ \pm} E\left(m_{1}\right)-\left[\left(a^{ \pm}\right)^{2}-m_{1}\right] \Pi\left(-a^{ \pm}, m_{1}\right)}{\left(a^{ \pm}+1\right)\left(a^{ \pm}+m_{1}\right) K\left(m_{1}\right)}, & -1 \mp 2 h \leqslant \varepsilon^{\prime} \leqslant h^{2} \\
1+\frac{E\left(m_{2}\right)-\Pi\left(a^{2}, \frac{m_{2}}{m_{2}-1}\right) / \sqrt{1-m_{2}}}{\left[m_{2}+a^{2} /\left(1-a^{2}\right)\right] K\left(m_{2}\right)}, & \varepsilon^{\prime} \geqslant h^{2},\end{cases}
\end{aligned}
$$

where $E(m)$ and $\Pi(\nu, m)$ are complete elliptic integrals of the second and third kinds, respectively.

Hence, noting that $W_{s t}$ is the equilibrium MaxwellBoltzmann distribution $W_{0}$, viz.,

$$
\begin{array}{r}
W_{0}[\phi(0), \dot{\phi}(0)] d \phi(0) d \dot{\phi}(0) \\
=\frac{\eta e^{-\eta^{2} \phi^{2}(0)+2 \sigma \cos ^{2} \phi(0)+4 \sigma h \cos \phi(0)}}{2 \sqrt{\pi^{3}} e^{\sigma} \sum_{m=-\infty}^{\infty} I_{m}(\sigma) I_{-2 m}(4 \sigma h)}
\end{array}
$$$$
\times d \phi(0) d \dot{\phi}(0)
$$

by making the transformation of the variables $\{\phi(0), \dot{\phi}(0)\}$ $\rightarrow\{w, \varepsilon\},{ }^{38}$ and by integrating the distribution function $W_{0}$ over the phase $w$, we obtain

$$
\begin{aligned}
W_{0}(\varepsilon) d \varepsilon= & \frac{e^{-\sigma-\varepsilon} d \varepsilon}{\pi^{3 / 2} \sum_{m=-\infty}^{\infty} I_{m}(\sigma) I_{-2 m}(4 \sigma h)} \\
& \times \begin{cases}K\left[m_{1}(\varepsilon)\right] / M_{1}(\varepsilon), & -1 \mp 2 h \leqslant \varepsilon^{\prime} \leqslant h^{2} \\
2 K\left[m_{2}(\varepsilon)\right] / M_{2}(\varepsilon), & \varepsilon^{\prime} \geqslant h^{2} .\end{cases}
\end{aligned}
$$

The average of a dynamical quantity $A(\varepsilon)$ is defined as

$$
\begin{aligned}
\left\langle\overline{\langle A\rangle_{0}=}\right. & \frac{e^{-\sigma}}{\pi^{3 / 2} \sum_{m=-\infty}^{\infty} I_{m}(\sigma) I_{-2 m}(4 \sigma h)} \\
& \times\left[\int_{-2 \sigma(1+2 h)}^{2 \sigma h^{2}}=\right. \\
& +\int_{-2 \sigma(\varepsilon) K\left[m_{1}(\varepsilon)\right] M_{1}^{-1}(\varepsilon) e^{-\varepsilon} d \varepsilon}^{2 \sigma h^{2}} A(\varepsilon) K\left[m_{1}(\varepsilon)\right] M_{1}^{-1}(\varepsilon) e^{-\varepsilon} d \varepsilon \\
& \left.+2 \int_{2 \sigma h^{2}}^{\infty} A(\varepsilon) K\left[m_{2}(\varepsilon)\right] M_{2}^{-1}(\varepsilon) e^{-\varepsilon} d \varepsilon\right] .
\end{aligned}
$$

In particular, one can verify that the equipartition theorem holds, viz.,

$$
\eta^{2}\left\langle\overline{\overline{\dot{\phi}^{2}}}\right\rangle_{0}=\left\langle\varepsilon+2 \sigma \overline{\overline{\cos ^{2} \phi}}+4 \sigma h \overline{\overline{\cos \phi}}\right\rangle_{0}=\frac{1}{2} .
$$

${ }^{1}$ H. Risken, The Fokker-Planck Equation, 2nd ed. (Springer, Berlin, 1989).

${ }^{2}$ W. T. Coffey, Yu. P. Kalmykov, and J. T. Waldron, The Langevin Equation, 2nd ed. (World Scientific, Singapore, 2004).

${ }^{3}$ G. Barone and A. Paterno, Physics and Applications of the Josephson Effect (Wiley, New York, 1982).

${ }^{4}$ W. T. Coffey, D. S. F. Crothers, Yu. P. Kalmykov, and J. T. Waldron, Phys. Rev. B 51, 15947 (1995).

${ }^{5}$ D. A. Garanin, Phys. Rev. E 54, 3250 (1996).

${ }^{6}$ W. T. Coffey, D. S. F. Crothers, and S. V. Titov, Physica A 298, 330 (2001).

${ }^{7}$ Yu. P. Kalmykov, Phys. Rev. E 61, 6320 (2000); 62, 2270 (2000).

${ }^{8}$ H. A. Kramers, Physica (Utrecht) 7, 284 (1940).

${ }^{9}$ P. Hänggi, P. Talkner, and M. Borkovec, Rev. Mod. Phys. 62, 251 (1990).

${ }^{10}$ W. T. Coffey, D. A. Garanin, and D. McCarthy, Adv. Chem. Phys. 117, 528 (2001).

${ }^{11}$ V. I. Mel'nikov, Physica A 130, 606 (1985);Phys. Rep. 209, 1 (1991).

${ }^{12}$ V. I. Mel'nikov and S. V. Meshkov, J. Chem. Phys. 85, 1018 (1986).

${ }^{13}$ H. Grabert, Phys. Rev. Lett. 61, 1683 (1988).

${ }^{14}$ E. Pollak, H. Grabert, and P. Hänggi, J. Chem. Phys. 91, 4073 (1989).

${ }^{15}$ I. Rips and E. Pollak, Phys. Rev. A 41, 5366 (1990).

${ }^{16}$ M. Topaler and N. Makri, J. Chem. Phys. 101, 7500 (1994).

${ }^{17}$ A. N. Drozdov and P. Talkner, J. Chem. Phys. 109, 2080 (1998).

${ }^{18}$ R. Ferrando, R. Spadacini, and G. E. Tommei, Phys. Rev. A 46, R699 (1992); Phys. Rev. E 48, 2437 (1993).

${ }^{19}$ E. Pollak, J. Bader, B. J. Berne, and P. Talkner, Phys. Rev. Lett. 70, 3299 (1993).

${ }^{20}$ Yu. Georgievskii and E. Pollak, Phys. Rev. Lett. 70, 5098 (1994).

${ }^{21}$ E. Hershkovitz, P. Talkner, E. Pollak, and Yu. Georgievskii, Surf. Sci. 421, 73 (1999).

${ }^{22}$ R. W. Pastor and A. Szabo, J. Chem. Phys. 97, 5098 (1992).

${ }^{23}$ W. T. Coffey, Yu. P. Kalmykov, and S. V. Titov, J. Chem. Phys. 120, 9199 (2004).

${ }^{24}$ P. M. Déjardin, D. S. F. Crothers, W. T. Coffey, and D. J. McCarthy, Phys. Rev. E 63, 021102 (2001).

${ }^{25}$ Y. P. Kalmykov, W. T. Coffey, B. Ouari, and S. V. Titov, J. Magn. Magn. Mater. 292, 372 (2005).

${ }^{26}$ W. T. Coffey, M. W. Evans, and P. Grigolini, Molecular Diffusion and Spectra (Wiley, New York, 1984).

${ }^{27}$ Handbook of Mathematical Functions, edited by M. Abramowitz and I. Stegun (Dover, New York, 1964).

${ }^{28}$ R. A. Sack, Proc. Phys. Soc. London, Sect. B 70, 402 (1957).

${ }^{29}$ A. Szabo, J. Chem. Phys. 72, 4620 (1980).

${ }^{30}$ J. I. Lauritzen and R. Zwanzig Jr., Adv. Mol. Relax. Processes 5, 339 (1973).

${ }^{31}$ E. Praestgaard and N. G. van Kampen, Mol. Phys. 43, 33 (1981)

${ }^{32}$ J. P. Poley, Appl. Sci. Res., Sect. B 4, 337 (1955).

${ }^{33}$ K. S. Gilroy and W. A. Philips, Philos. Mag. B 43, 735 (1981).

${ }^{34}$ W. T. Coffey, D. S. F. Crothers, and Yu. P. Kalmykov, Phys. Rev. E 55, 4812 (1997). 
${ }^{35}$ H. T. Davis, Introduction to Nonlinear Differential and Integral Equations (Dover, New York, 1962).

${ }^{36}$ E. T. Whittaker and G. N. Watson, A Course of Modern Analysis, 4th ed. (Cambridge University Press, Cambridge, England, 1927).
${ }^{37}$ A. Prudnikov, Yu. A. Brychkov, and O. I. Marichev, Integrals and Series, More Special Functions (Gordon and Breach, New York, 1990), Vol. 3.

${ }^{38}$ Yu. P. Kalmykov, Khim. Fiz. 6, 745 (1987) [Sov. J. Chem. Phys. 6, 1427 (1990). 